\title{
Antimicrobial and Antioxidant Effects of Nerium oleander Flower Extracts
}

\author{
S. Saranya, D. Archana and K.S. Santhy* \\ Department of Zoology, Avinashilingam University, (Institute for Home Science and \\ Higher Education for Women), Coimbatore, Tamil Nadu, India \\ *Corresponding author
}

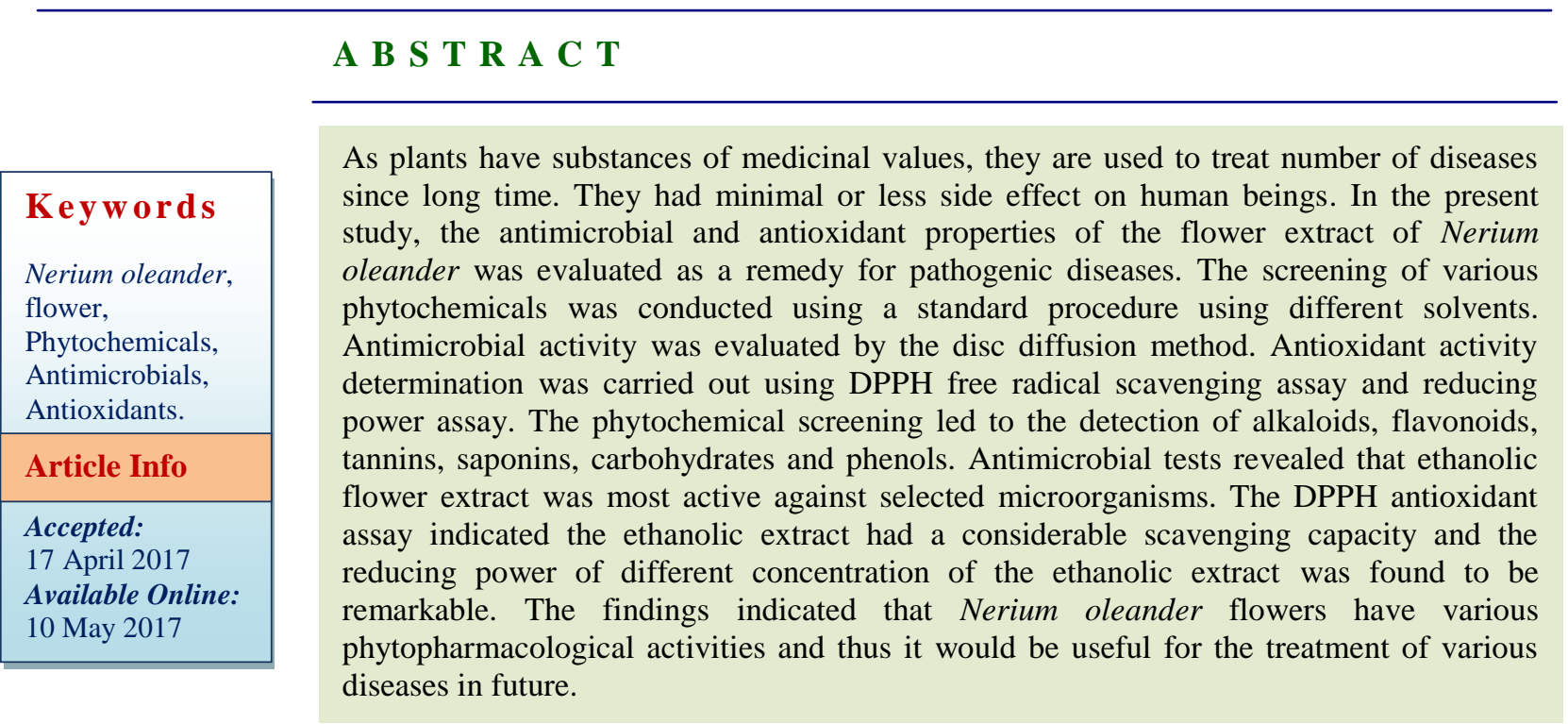

\section{Introduction}

From the beginning of human civilization, plant and plant products are usually used to treat different diseases (Joshi et al., 2009). Researchers have great interest in those substances which are derived from plants because they are versatile in their applications.

Various phytochemicals can be obtained from plants which are very beneficial for mankind and medicinal plants have become the richest biological resource of such chemicals which are used in manufacturing of traditional drugs as well as in modern nutraceuticals, food supplements, medicines, folk medicines, raw material and pharmaceutical intermediates for synthetic drugs (Tumwine, 2011).

Nerium oleander (Family: Apocyanacea) is a beautiful free flower especially suited to sunny and dry localities (Lokesh et al., 2010). Flowers are the most attractive part of the plant. These are rich in color and sweet fragrance. Not only humans, but the animals and the insects all gets attracted to it. Honey bee sucks nectar from the flowers; by which natural honey is prepared. Flowers are used to show different emotions; happiness, grief, sadness, lost, celebrations and many more. Red flowers are mostly used to denote love 
and affection. Most of the flowers occupy the potency as they are used in different medicinal fields. In Chinese therapies, Ayurveda and Naturopathy flowers plays a major role for the treatment of many diseases.

Globally, researchers are using extracts of plants for their antiviral, antibacterial, and antifungal activities. The characteristics of the plants that retard the growth of microorganisms have been investigated in different laboratories around the world since 1926 (Bakht et al., 2012). Hence, in the present investigation, same efforts are continued in the progression of searching novel therapeutics against antibiotic activity.

Recently there has been an upsurge of interest in the therapeutic potential of medicinal plants as antioxidants in reducing oxidative stress-induced tissue injury (Pourmorad et al., 2006). Among the numerous naturally occurring antioxidants; ascorbic acid, carotenoids and phenolic compounds are more effective (Duh et al., 1999). They are known to inhibit lipid peroxidation (by inactivating lipoxygenase), to scavenge free radicals and active oxygen species by propagating a reaction cycle and to chelate heavy metal ions.

The studies carried out on medicinal plants and vegetables strongly support the idea that plant constituents with antioxidant activity are capable of exerting protective effects against oxidative stress in biological systems. In the present investigation, we studied flower extracts of Nerium oleander for the search of natural and novel antioxidants.

\section{Materials and Methods}

\section{Collection and authentication of the plant}

Nerium oleander flowers were collected in December 2016 from the campus of
Avinashilingam University (Institute for Home Science and Higher Education for Women), Coimbatore. The whole plant was identified (BSI/SRC/5/23/2017/Tech/3265) and authenticity was confirmed by Dr. C. Murugan, Scientist D, Botanical survey of India, T.N.A.U Campus, Coimbatore

\section{Preparation of the flower extract}

The flowers were cleaned thoroughly and dried at room temperature for 5-7 days in the shade. The dried samples were powdered using an electrical grinder. The powdered samples were stored in screw cap bottles until further analysis.

Five hundred grams of powder was taken, to which $50 \mathrm{ml}$ of different solvents (ethanol, chloroform and water) were added, mixed, and kept for four days. The contents were periodically shaken using an electric shaker. After four days, the contents were filtered through a Buchner funnel in a conical flask and it was further concentrated by evaporation by keeping the filtrate in a roundbottomed flask, till the solvent completely evaporated and the extract settled down to the bottom.

\section{Preliminary phytochemical screening}

Preliminary screening of the extracts and identification of major phytochemical was done by color tests adapting standard methods by Raman (2006).

\section{Antimicrobial activity}

\section{Test organisms}

Two Gram-positive bacteria (Bacillus subtilis, Staphylococcus aureus) and three Gramnegative bacteria (Escherichia coli, Salmonella typhi and Pseudomonas aeruginosa) were used for antibacterial 
activity. Four fungi (Aspergillus niger, Aspergillus fumigatus, Aspergillus flavus, Aspergillus rhizopus) were used for antifungal activity.

\section{Methodology}

Antibacterial and antifungal activity studies were carried out by agar diffusion method (Barry et al., 1976). Standard antibiotic disc of Chloramphenicol (K-30 $\mu \mathrm{g} / \mathrm{disc})$ was used as the standard reference drug for antibacterial assay, Nystatin $(50 \mu \mathrm{g} / \mathrm{disc})$ was used for antifungal activity study (Sarkar et al., 1998).

The pure cultures of different pathogens were grown overnight in sterile nutrient broth and incubated at $37^{\circ} \mathrm{C}$ for 24 hours. The $0.1 \mathrm{ml}$ of the culture was seeded on $25 \mathrm{ml}$ of solidified nutrient agar and rose bengal plates for bacterial and fungal cultures, respectively. The wells were bored with $8 \mathrm{~mm}$ borer in seeded agar, and then the particular concentrations $(20 \mu \mathrm{l})$ of the extracts were added in each well. Soon after the plates were then kept at $10^{\circ} \mathrm{C}$ for $30 \mathrm{~min}$. After it normalized to room temperature plates were incubated at $37^{\circ} \mathrm{C}$ for $24 \mathrm{hrs}$. After incubation period is completed, the zone of inhibition was measured and recorded.

\section{Antioxidant studies}

\section{DPPH free radical scavenging activity (Mensor et al., 2001)}

1, 1-Diphenyl-2-picrylhydrazyl (DPPH) was obtained from Sigma Aldrich Co., St. Louis, USA. The diluted working solutions of the test extracts were prepared in ethanol. Ascorbic acid was used as standard in 5$30 \mu \mathrm{g} / \mathrm{ml}$ solution. $0.002 \%$ of DPPH was prepared in ethanol and $1 \mathrm{ml}$ of this solution was mixed with $1 \mathrm{ml}$ of sample solution and standard solution separately. These solution mixtures were kept in dark for $30 \mathrm{~min}$ and optical density was measured at $517 \mathrm{~nm}$ using UV spectrophotometer. Ethanol $(1 \mathrm{ml})$ with DPPH solution $(0.002 \%, 1 \mathrm{ml})$ was used as blank. The optical density was recorded and $\%$ inhibition was calculated using the formula given below:

Percent $(\%)$ inhibition of DPPH activity = 100- (A-B/A) x100

Where A = optical density of the blank and B $=$ optical density of the sample.

\section{Reducing power assay (Oyaizu, 1986)}

Reaction mixtures were prepared by adding $2.5 \mathrm{ml}$ of phosphate buffer $(0.2 \mathrm{M}, \mathrm{pH} 6.6)$, $2.5 \mathrm{ml}$ potassium ferricyanide $(1 \%)$ and varying concentrations of extracts (5$30 \mu \mathrm{g} / \mathrm{ml}$ ). After, the reaction mixtures were incubated at $50^{\circ} \mathrm{C}$ in water bath for $30 \mathrm{~min}$, allowed to cool at room temperature $\left(28^{\circ} \mathrm{C}\right)$, and $2.5 \mathrm{ml}$ of $10 \%$ TCA (Trichloro acetic acid) were added to each reaction mixture, and then centrifuged at $2000 \mathrm{rpm}$ for $10 \mathrm{~min}$. The supernatant $(2.5 \mathrm{ml})$ was separated in the test tube and added with $2.5 \mathrm{ml}$ of distilled water and $0.5 \mathrm{ml} \mathrm{FeCl}_{3}(1.0 \%)$, and allowed to react for $10 \mathrm{~min}$ at room temperature and the absorbance was measured at $700 \mathrm{~nm}$. Ascorbic acid solution was used as standard.

\section{Results and Discussion}

\section{Preliminary phytochemical screening of Nerium oleander flower}

The phytochemical constituents serve as therapeutic agents as well as important raw materials for the manufacture of traditional and modern medicine. Investigations on secondary plant constituents have made phenomenal advance during the past few decades. Based on the above concept few analysis were done with the extracts were described below (Table 1). 


\section{Antimicrobial activity}

\section{Antibacterial activity of flower extract of Nerium oleander}

The flower extracts were evaluated for its antibacterial activity against five clinical bacterial isolates namely Bacillus subtilis, Staphylococcus aureus, Escherichia coli, Salmonella and Pseudomonas aeruginosa. Table 2 described the antibacterial activity of ethanol, chloroform and water extracts of the Nerium oleander against these bacterial isolates.

From the table it was observed that the zone of inhibition was found to be maximum in the ethanol extract and was found to be more active against Pseudomonas aeruginosa (28mm) Salmonella (25mm) Staphylococcus aureus $(21 \mathrm{~mm})$, Escherichia coli $(20 \mathrm{~mm})$ followed by Bacillus subtilis $(17 \mathrm{~mm})$. Aqueous extract showed moderate activity and chloroform extract had minimal activity against the tested micro organisms.

\section{Antifungal activity of flower extracts of Nerium oleander}

The antifungal activity of the Nerium oleander flower extract was determined against the fungal isolates namely Aspergillus niger, Aspergillus flavus, Aspergillus fumigates and Rhizopus species. Table 3 depicts the antifungal activity of Nerium oleander flower extracts.

Here also the ethanol extract showed the maximum activity when compared to other extracts. Maximum zone of inhibition was reported against Aspergillus flavus (18mm) Rhizopus (18mm) whereas the minimum activity was reported against Aspergillus fumigates $(17 \mathrm{~mm})$ and Aspergillus niger $(13 \mathrm{~mm})$.

\section{Antioxidant activity}

\section{DPPH radical scavenging activity}

The results of the assay are expressed in scavenging activity of DPPH free radical expressed in percentage. The DPPH assay of ethanol extract of Nerium oleander and the reference compound ascorbic acid is given in figure 1.

\section{Reducing power ability of Nerium oleander flower extracts}

The reducing power of different concentration of Nerium oleander was found to be remarkable and the absorbance of each concentration was found to rise as the concentration gradually increases. Compounds with reducing power indicate that they are electron donors and can reduce the oxidized intermediates of free radical reactions, so that they can act as primary and secondary antioxidants. From the graph (Fig. 2) it is clear that as the absorbance of the extracts increased, the reducing power ability also increased suggesting the presence of electron donors in the extract which act as intermediates for radical scavenging reactions.

Use of flowers as a source of medicine has been inherited and is an important component of the health care system in India. Flower extracts are given singly of as concoctions for various ailments. Many investigations have demonstrated to elucidate the chemical components of flower origin. In the present study, phytochemical, antimicrobial and antioxidant activities of the flower extract was carried out to find out the major activities.

The phytochemical screening and qualitative estimation of the plant studies showed that the flowers were rich in alkaloids, flavanoids, phenols and triterpenoids in all the extracts. 
Some extract showed presence of carbohydrates and sterols too. Saponins and tannins were found to be present in all the extracts of the flower. It should be noted that steroidal compounds are of importance and of interest in pharmacy due to their relationship with sex hormones.

Flavonoids are polyphenolic compounds and consist of flavones, flavonols, flavanols, flavanone and flavanonols. These compounds represent the majority of plant secondary metabolites and have shown to possess remarkable health promotory effects such as anti-inflammatory, antioxidant, antimicrobial and anticancer properties. Interception of free radicals or other reactive species is mainly by radical scavenging and is caused by various antioxidants like vitamin $\mathrm{C}$ and $\mathrm{E}$, glutathione, other thiol compounds, carotenoids, flavonoids, etc.

All the plant extracts used in this study were primarily screened against the tested microorganisms by agar well diffusion method. According to the World Health Organization (2012) the evolving public health threat of antimicrobial resistance is driven by both appropriate and inappropriate use of anti-infective medicines. The development of bacterial resistance to presently available antibiotics has necessitated the need to search for new antibacterial agents. Different antibiotics exercise their inhibitory activity on different pathogenic organisms (Chanda and Rakholiya, 2011).

Table.1 Preliminary phytochemical analysis of flower extracts of Nerium oleander

\begin{tabular}{|c|c|c|c|c|c|}
\hline \multirow{2}{*}{ S. No. } & \multirow{2}{*}{ Constituents } & \multirow{2}{*}{ Test for constituents } & \multicolumn{3}{|c|}{ Solvents } \\
\hline & & & Ethanol & Chloroform & Aqueous \\
\hline \multirow{3}{*}{1} & \multirow{3}{*}{ Alkaloids } & Mayers & + & + & + \\
\hline & & Wagners & + & - & + \\
\hline & & Dragendroffs & + & + & _- \\
\hline \multirow{2}{*}{2} & \multirow{2}{*}{ Flavonoids } & Alkaline reagent & + & + & _- \\
\hline & & Lead acetate test & + & - & _ \\
\hline \multirow{2}{*}{3} & \multirow{2}{*}{ Sterols } & Libermann Burchard & - & - & _ \\
\hline & & Salkowski's & + & - & _- \\
\hline \multirow{2}{*}{4} & \multirow{2}{*}{ Phenols } & Ferric chloride & - & + & + \\
\hline & & Lead acetate & + & - & __ \\
\hline 5 & Saponins & Foam test & + & + & + \\
\hline 6 & Tannins & Gelatin test & + & + & + \\
\hline 7 & Quinones & Alcoholic $\mathrm{KOH}$ & _- & - & _- \\
\hline \multirow{2}{*}{8} & \multirow{2}{*}{ Proteins } & Ninhydrin & + & - & - \\
\hline & & Biuret test & + & - & + \\
\hline \multirow{2}{*}{9} & \multirow{2}{*}{ Carbohydrates } & Molisch's test & + & + & _ \\
\hline & & Fehling's test & + & + & + \\
\hline
\end{tabular}


Table.2 Antibacterial activity of the flower extract of Nerium oleander

\begin{tabular}{|l|l|l|l|l|}
\hline \multirow{2}{*}{ Bacterial isolates } & \multicolumn{4}{|l|}{ Zone of inhibition in diameter $(\mathrm{mm})$} \\
\cline { 2 - 5 } & ETA & CHL & AQE & Control* $^{*}$ \\
\hline Bacillus subtilis & $17 \pm 0.5$ & $9 \pm 0.2$ & $15 \pm 0.5$ & $25 \pm 1.5$ \\
\hline Escherichia coli & $20 \pm 1.5$ & $10 \pm 0.5$ & $22 \pm 1.5$ & $30 \pm 2.5$ \\
\hline Pseudomonas aeruginosa & $28 \pm 2.6$ & $18 \pm 1.5$ & $25 \pm 1.7$ & $36 \pm 3.6$ \\
\hline Staphylococcus aureus & $21 \pm 1.2$ & $12 \pm 1.5$ & $10 \pm 0.5$ & $35 \pm 2.5$ \\
\hline Salmonella typhii & $25 \pm 3.0$ & $15 \pm 1.5$ & $20 \pm 1.5$ & $30 \pm 3.0$ \\
\hline
\end{tabular}

All values are expressed as Mean \pm Standard deviation of four replicates

ETA - Ethanol extract, CHL - Chloroform extract, AQE - Aqueous extract, *Control - Chloramphenicol

Table.3 Antifungal activity of the flower extracts of Nerium oleander

\begin{tabular}{|l|l|l|l|l|}
\hline \multirow{2}{*}{ Fungal isolates } & \multicolumn{4}{|l|}{ Zone of inhibition in diameter (mm) } \\
\cline { 2 - 5 } & ETA & CHL & AQE & Control* \\
\hline Aspergillus niger & $13 \pm 0.5$ & $15 \pm 1.3$ & $14 \pm 1.2$ & $15 \pm 0.5$ \\
\hline Aspergillus flavus & $18 \pm 1.5$ & $17 \pm 1.5$ & $18 \pm 1.5$ & $13 \pm 1.5$ \\
\hline Aspergillus fumigatus & $17 \pm 0.5$ & $16 \pm 1.5$ & $13 \pm 0.5$ & $15 \pm 0.5$ \\
\hline Rhizopus & $18 \pm 1.5$ & $17 \pm 0.5$ & $10 \pm 0.5$ & $17 \pm 0.5$ \\
\hline
\end{tabular}

All values are expressed as Mean \pm Standard deviation of four replicates

ETA - Ethanol extract, CHL - Chloroform extract, AQE - Aqueous extract, *Control - Nystatin

Fig.1 DPPH radical scavenging activity of Nerium oleander flower

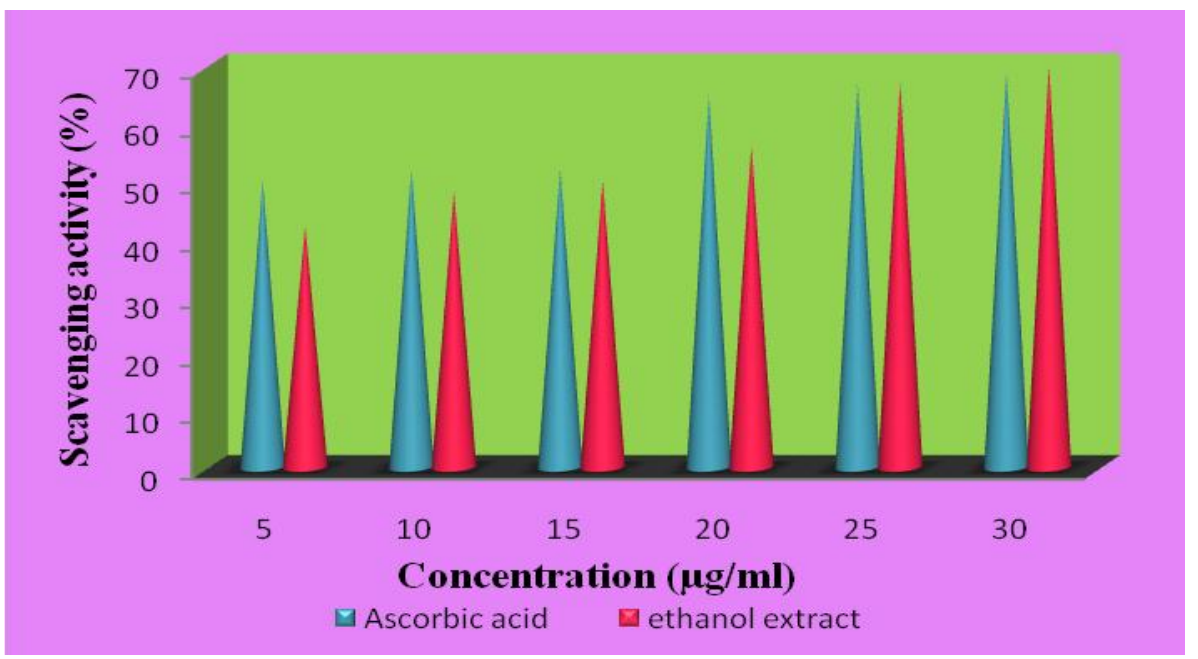


Fig.2 Reducing power ability of Nerium oleander flower extract

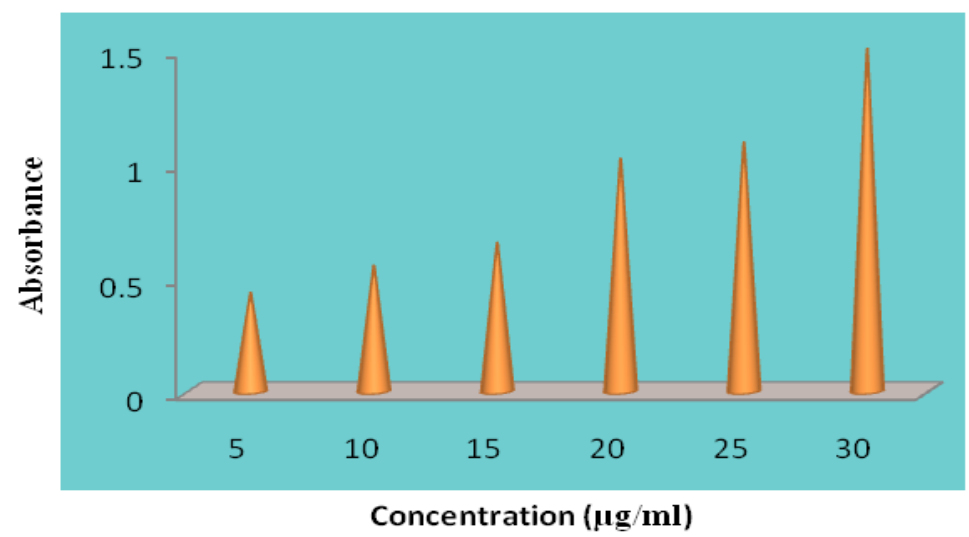

In the present investigation, different solvents of flower extract showed varying activity against gram positive and gram negative bacteria. Maximal activity of the ethanol extract points out that the active components present in ethanol flower extract can prove to be a great remedy for treating diseases. The mean inhibition zone for the tested bacteria ranged from $(9 \mathrm{~mm}-28 \mathrm{~mm})$ indicating a remarkable antibacterial effect when compared with Chloramphenicol the positive control, which ranged from $25 \mathrm{~mm}-36 \mathrm{~mm}$.

Similar results were reported by Jeyachandran et al., (2010) in which the ethanolic extract of Nerium oleander showed maximum zone of inhibition $(28 \mathrm{~mm})$ against Salmonella typhi. Also Aboud (2015) reported that ethanol extracts of Nerium oleander showed highest activity other than aqueous extract. The highest activity was demonstrated by the ethanol extract against Staphylococcus aureus and Klebsiella spp

The efficacy of ethanol extract of flowers of Nerium oleander demonstrated the presence of cell wall active antifungal agents which could lead to the discovery and development of novel antifungal treatment therapies. Similarly such results were documented by Nitave and Patil (2015) who reported the antifungal activity of the ethanolic flower extracts against the Ciprofloxacin standard. Since antiquity, natural products, especially those of plant origin have always been an important source of therapeutic agents. Recent data from the pharmaceutical industry show that natural products represent a valuable source for the production of new chemical entities. Indeed, Reactive Oxygen Species (ROS) released by the human body are eliminated by molecules with antioxidant properties.

The DPPH radical has been extensively used to evaluate the reducing substances and is a useful reagent for investigating the free radical scavenging activities of compounds. The radical scavenging activity of the ethanol extract from Nerium oleander flowers and ascorbic acid at different concentrations was tested by DPPH method and the results showed relatively high DPPH scavenging activity comparing with those extracts from other parts of Nerium oleander.

Different studies have indicated that the reducing capacity of bioactive compounds is associated with its antioxidant activity (Siddhuraju et al., 2002). In this study, the reducing power of Nerium oleander extract was determined. The extracts showed some degree of electron donation capacity in a concentrationdependent manner, but the capacities were lower than that of ascorbic acid.

\section{Acknowledgements}

The authors wish to place their record of thanks to the Department of Zoology, Avinashilingam University (Institute for Home Science and 
Higher education for Women), Coimbatore, Tamil Nadu for providing infrastructure facility.

\section{References}

Aboud, A.S. 2015. Antimicrobial activities of aqueous and ethanolic extracts from Nerium oleander used in the treatment of burns infections isolates. J. Pharm. Chem. Biol. Sci., 2(4): 248-258.

Bakht, J., G. Azra and Shafi, M. 2012. Antimicrobial activity of Nicotiana tabacum using different solvents extracts. Pak. J. Bot., 44(1): 459 - 463.

Barry, A.L. 1976. Principle and Practice of Microbiology Lea \& Fabager. Philadelphia.

Chanda, S. and Rakholiya, K. 2011. Combination therapy: Synergism between natural plant extracts and antibiotics against infectious diseases. Science against microbial pathogens: communicating current research and technological advances. A. MéndezVilas (Ed.). pp. 520-529.

Duh, P.D., Y.Y. Tu and Yen, G.C. 1999. Antioxidant activity of aqueous extract of Harnjyur (Chrysanthemum morifolium Ramat). Lebensmwiss Technol., 32: 269 277.

Jeyachandran, R., X. Baskaran and Cindrella, L. 2010. Screening of phytochemical and antibacterial potential of four Indian medicinal plants. Libyan Agri. Res. Center J. Int., 1(5): 301-306.

Joshi, B., B. Sunil and Anuja S. 2009. Antibacterial property of different medicinal plants: Ocimum sanctum, Cinnamomum zeylanicum, Xanthoxylum armatum and Origanum majorana. Kathmandu University J. Sci. Engi. Technol., 5(1): 143-150.

Lokesh, R., E. Leonard Barnaba, P. Madhuri, K. Saurav and Sundar K. 2010. Larvicidal activity of Trigonella foenum and Nerium oleander leaves against mosquito larvae found in Vellore city. India, Curr. Res. J. Biol. Sci., 3: 154 - 160.

Mensor, L.L., F.S. Menezes, G.G. Leitao, A.S. Reis, T.S. Santos and Coube, C.S. 2001. Screening of Brazilian plant extracts for antioxidant activity by the use of DPPH free radical method. Phytother. Res., 15: 127130.

Nitave, S.A. and Patil, V.A. 2015. Study of antibacterial and antifungal activity of Nerium oleander flower extract and its phytochemical screening. World $J$. Pharmaceutical Res., 5(1): 640 - 647.

Oyaizu, M. 1986. Studies on product of browning reaction prepared from glucose amine. $J$. Nutr., 44: 307-15.

Pourmorad, F., S.J. Hosseinimehr and Shahabimajd, N. 2006. Antioxidant activity, phenols, flavanoid contents of selected Iranian medicinal plants. $S . \quad A f r . \quad J$. Biotechnol., 5: 1142-1145.

Raman, N. 2006. Phytochemical Technique. New Indian Publishing Agencies: New Delhi, pp.19.

Sarkar, M.K., D. Ergil, A.U. Tamer and Sahin, N. 1998. Antiamoebic and spasmolytic activities of extracts from some antidiarrhoeal traditional preparations used in Kinshasa Fitoterapia. 69: 457.

Siddhuraju, P.P., S. Mohan and Becker, K. 2002. Studies on the antioxidant activity of Indian laburnum (Cassia fistula L.): a preliminary assessment of crude extracts from stem bark, leaves, flowers and fruit pulp. Food Chem., 79: 61 - 67.

Tumwine, W. 2011. Implementation of the framework convention on tobacco control in Africa: Current Status of Legislation. Int. J. Environ. Res. Public Health, 8: 4312-4331.

World Health Organization. 2012. The evolving threat of antimicrobial resistance options for action, ISBN: 9789241503181.

\section{How to cite this article:}

Saranya, S., D. Archana and Santhy, K.S. 2017. Antimicrobial and Antioxidant Effects of Nerium oleander Flower Extracts. Int.J.Curr.Microbiol.App.Sci. 6(5): 1630-1637.

doi: https://doi.org/10.20546/ijcmas.2017.605.178 